\title{
Consumo alimentar e avaliação nutricional: caracterização de escolares no município de Maringá-Paraná
} Food consumption and nutritional evaluation: school characterization in Maringá - Paraná

Suelen Dayane Pereira Cadamuro', Daniel Vicentini de Oliveira², Rose Mari Bennemann', Eraldo Schunk Silva ${ }^{3}$, José Eduardo Gonçalves ${ }^{1}$

'Centro Universitário Cesumar (UNICESUMAR), Maringá, PR, Brasil.

ZUniversidade Estadual de Campinas (UNICAMP), Campinas, SP, Brasil.

3Universidade Estadual de Maringá (UEM), Maringá, PR, Brasil.

Recebido em: 27/06/2016 / Aceito em: 30/06/2016

d.vicentini@hotmail.com

\section{RESUMO}

Objetivo: avaliar o estado nutricional dos escolares com relação ao consumo alimentar. Método: estudo transversal, realizado com 82 crianças de 5 a 7 anos, matriculadas em quatro escolas municipais de Maringá, estado do Paraná. Para a avaliação nutricional foi aferida a estatura e o peso atual dos escolares. Utilizou-se o cálculo do índice de massa corporal (IMC) e os escolares foram classificados conforme o IMC e por idade em escore-z do sistema de vigilância alimentar e nutricional. Foi aplicado aos pais o questionário de consumo alimentar do sistema de vigilância alimentar nutricional. Resultados: a proporção de crianças do sexo feminino foi de $48,78 \%$ e $51,21 \%$ do sexo masculino. A maioria encontrava-se com o IMC adequado (78.04\%); $21,95 \%$ dos escolares estavam acima do peso. A frequência do consumo alimentar demonstrou que 74,37\% consumiam alimentos saudáveis mais de três vezes na semana e para os alimentos não saudáveis, $58,53 \%$ das crianças consumem até três vezes na semana. Considerações finais: conclui-se que a maior parte das crianças avaliadas da rede municipal de ensino possuem IMC adequado e consomem alimentos saudáveis na maioria dos dias da semana, o que pode ser fator protetor para obesidade nesta população. Porém, há um percentual considerável acima do peso, o que requer cuidados e orientações especiais da escola e órgãos públicos de saúde e educação.

Palavras-chave: Infância; Nutrição; Obesidade; Saúde.

\section{ABSTRACT}

Objective: evaluate the nutritional status of students with respect to food consumption. Method: cross-sectional study involving 82 children aged 5 to 7, enrolled in four public schools in Maringá, Paraná, Brazil. We measured weight and height of subjects and calculated the body mass index (BMI). Students were classified according to BMI and z-score of the food and nutrition surveillance system. We applied the questionnaire of food intake of nutritional food surveillance system in all subjects parents. Results: proportion of female children was $48.78 \%$ and $51.21 \%$ male, where the majority was in with the appropriate BMI (78.04\%); $21.95 \%$ of students were overweight. The frequency of food intake showed that $74.37 \%$ consumed healthy food more than three times a week and for the unhealthy foods, $58.53 \%$ of children consume up to three times a week. Closing remarks: we conclude that most schoolchildren of the municipal school system have adequate BMI and consume healthy food on most days of the week, which may be a protective factor for obesity in this population. However, there is a considerable overweight percentage, which requires special care and guidance of school and public health agencies and education.

Keywords: Childhood; Nutrition; Obesity; Health. 


\section{INTRODUÇÃO}

Nos últimos anos, numerosos estudos têm sido realizados, relacionados à obesidade infantil. Segundo a Organização Mundial da Saúde, ${ }^{1}$ a obesidade é uma doença em que o excesso de gordura corporal acumulada pode afetar a saúde. $O$ excesso de peso e obesidade no Brasil tem sido encontrado em escolares, a partir dos cinco anos de idade, em todas as regiões e classes sociais. ${ }^{2}$ Além disso, a obesidade infantil interfere na saúde cardiovascular e metabólica. ${ }^{3}$

Crianças obesas apresentam, frequentemente, baixa autoestima, afetando o desempenho escolar e relacionamentos sociais, podendo incorrer em consequências psicológicas mais graves a longo prazo. ${ }^{4}$ As meninas obesas podem entrar antes na puberdade, ter ciclos menstruais irregulares; problemas cutâneos e metabólicos. ${ }^{5}$

Possivelmente os fatores comportamentais, tais como o estilo de vida sedentário e o excessivo consumo de alimentos calóricos, ${ }^{6}$ colaboram para o aumento da obesidade. Nesse contexto, acredita-se que o tempo dedicado assistindo à televisão possa ser um marcador para identificação de baixos níveis de atividade física e também de práticas alimentares não saudáveis.

Nos escolares, ${ }^{7}$ o elevado consumo de açúcar, gordura e pouco consumo de fibras associado a uma diminuição de atividade física, seria um importante fator de risco para a obesidade. Em uma revisão sistemática ${ }^{8}$ foi evidenciado que crianças em idade escolar ou jovens com excesso de peso têm mais risco de se tornarem adultos obesos ou de permanecerem com excesso de peso.

A adoção de bons hábitos alimentares desde a infância contribui para uma vida adulta mais saudável, ${ }^{9}$ mesmo sabendo que a mudança do padrão alimentar em nosso dia a dia está associada a um grande apelo comercial e um número crescente de oferta de alimentos industrializados com alta densidade energética, fonte de gordura saturada e carboidratos simples. ${ }^{10}$

Considerando a dificuldade do tratamento da obesidade, principalmente em escolares, é de fundamental importância à identificação de estratégias efetivas para sua prevenção. Assim o objetivo deste estudo é avaliar o estado nutricional dos escolares da cidade Maringá - Paraná com relação ao consumo alimentar realizado em sua casa.

\section{MÉTODO}

Participaram do estudo escolares com idade $\geq 5$ a $\leq 7$ anos, de ambos os sexos, pertencentes a quatro escolas da rede municipal de ensino de Maringá - Paraná, sendo duas pré-escolas e duas do ensino fundamental 1. Este estudo foi transversal, com coleta de dados primários e secundários.

O projeto teve aprovação do Comitê de ética em pesquisa da Unicesumar (parecer $n^{\circ} 182.935$ ). Todos os participantes foram devidamente esclarecidos sobre o objetivo da pesquisa e de forma voluntária assinaram o Termo de Consentimento Livre e Esclarecido antes de sua inclusão.

Neste trabalho como critério de exclusão, somente foram utilizados dados de escolares sem históricos de alergias alimentares, ou que faziam uso de medicamentos e/ou que estavam em tratamento médico. Estas informações foram coletadas na secretaria das escolas e também junto a suas famílias.

Para realização da avaliação antropométrica (peso e altura), utilizou-se balança eletrônica Plenna ${ }^{\circledR}$ e fita antropométrica Sanny ${ }^{\circledR}$ com capacidade de 2 metros. As medidas foram computadas para a obtenção do Índice de Massa Corporal (IMC) através da formula: peso (kg) / estatura $\left(\mathrm{m}^{2}\right)$.

Para classificação do estado nutricional dos avaliados, os resultados do IMC por idade foram comparados com a distribuição em escore-z da Organização Mundial da Saúde. ${ }^{11}$ Os valores obtidos foram classificados, segundo a tabela preconizada pelo Sistema de Vigilância Alimentar e Nutricional. ${ }^{12}$ Para melhor análise dos resultados, classificamos os escolares acima do peso, os escolares com risco para sobrepeso, sobrepeso e obesidade.

O consumo alimentar dos escolares foi avaliado pelo questionário de frequência alimentar que foi entregue aos pais durante as reuniões pedagógicas que ocorreram no inicio do ano letivo, momento em que os pais responderam sobre as preferências alimentares e a frequência de consumo dos alimentos citados em suas respectivas casas. ${ }^{12}$

Para análise do consumo dos escolares, os alimentos foram classificados em alimentos saudáveis e não saudáveis. Para os alimentos saudáveis incluem a salada crua, legumes e verduras cozidos, frutas frescas ou salada de frutas, feijão, leite e derivados. Os alimentos não saudáveis foram incluídos a batata frita, batata de pacote e salgados fritos; hambúrguer e embutidos; bolachas e biscoitos salgados ou salgadinhos de pacote; bolachas, biscoito doces ou recheados, doces, balas e chocolates; refrigerantes. ${ }^{12}$

Os resultados obtidos foram correlacionados pela comparação do consumo de alimentos com o IMC, através de tabelas de frequência simples e cruzada. O teste de Fisher foi utilizado para realizar a comparação entre o hábito de consumo de alimentos saudáveis e não-saudáveis, em relação às classes de consumo. Para esta análise considerou-se o nível de confiança de $95 \%$ ( $\alpha=0,05$ ), ou seja, $p$-valor menor que 0,05. Os dados foram analisados ${ }^{13,14}$ no Programa Statistical Analysis Software - SAS, version 9.0.

\section{RESULTADOS}

Participaram do estudo 82 escolares, sendo 42 (52\%) do sexo masculino e 40 (48\%) do sexo feminino. Com média de idade de 65 meses, que equivalem a 5 anos e 4 meses.

Para o estado nutricional, de ambos os sexos, (Tabela 1), em relação ao IMC, 64 (78,21\%) estão adequados, $8(9,58 \%)$ crianças estão com risco para sobrepeso, para sobrepeso num total de $4(4,94)$ crianças, para obesidade $6(7,26 \%)$ crianças no total.

Nas análises de consumo dos alimentos saudáveis (Tabela 2), das crianças com o IMC adequado, $52(63,41 \%)$ comem acima de três vezes na semana alimentos saudáveis e 12 (14,63\%) comem menos de três vezes na semana. Para as crianças acima do peso, 
Tabela 1 - Distribuição dos escolares segundo o estado nutricional.

\begin{tabular}{lcccc}
\hline & \multicolumn{2}{c}{ Feminino } & \multicolumn{2}{c}{ Masculino } \\
\hline IMC & $\mathbf{N}$ & $\%$ & $\mathbf{N}$ & $\%$ \\
\hline Adequado & 34 & 85,00 & 30 & 71,43 \\
Risco de sobrepeso & 1 & 2,50 & 7 & 16,67 \\
Sobrepeso & 3 & 7,50 & 1 & 2,38 \\
Obesidade & 2 & 5,00 & 4 & 9,52 \\
Total & 40 & 100,00 & 42 & 100,00 \\
\hline
\end{tabular}

$9(10,97 \%)$ comem acima de três vezes na semana e 8 $(9,76 \%)$ comem ate três vezes na semana, em um total de 82 crianças participantes da pesquisa.

Para os alimentos não saudáveis as crianças com peso adequado, $44(54,66 \%)$ consomem até três vezes na semana e $19(23,17 \%)$ consomem acima de três vezes na semana. Das crianças acima do peso, $13(15,85 \%)$ consomem até três vezes na semana e 3 $(3,67 \%)$ consomem acima de três vezes na semana, como mostra a Tabela 2.

Tabela 2 - Frequência semanal de consumo de alimentos saudáveis e não saudáveis por IMC, escolares, Maringá (PR), 2014

\begin{tabular}{lccccc}
\hline & \multicolumn{6}{c}{ IMC } \\
\cline { 2 - 6 } Consumo & Acima do peso & \multicolumn{1}{c}{ Adequado } & \\
\cline { 2 - 7 } & $\mathbf{N}$ & $\%$ & $\mathbf{N}$ & $\%$ & \\
\hline Alimentos saudáveis & & & & & \\
Até 3 vezes & 8 & 47,06 & 12 & 18,75 & \\
Acima de 3 vezes & 9 & 52,94 & 52 & 81,25 & $0,0257^{*}$ \\
Total & 17 & 100,00 & 64 & 100,00 & \\
\hline Alimentos não saudáveis & & & & & \\
Até 3 vezes & 13 & 81,25 & 44 & 69,84 & \\
Acima de 3 vezes & 3 & 18,75 & 19 & 31,16 & $0,5348^{*}$ \\
Total & 16 & 100,00 & 63 & 100,00 & \\
\hline
\end{tabular}

* Teste Exato de Fisher: nível de confiança de 95\% ( $\alpha=0,05)$, ou seja, $p$-valor menor que 0,05 .

\section{DISCUSSÃO}

A avaliação do estado nutricional em populações ${ }^{15}$ ainda está baseada predominantemente no índice de massa corporal (IMC) e este, por sua vez, continua sendo o utilizado no âmbito epidemiológico para esta análise. ${ }^{16} \mathrm{Em}$ nosso estudo, foram encontrados escolares com alteração do estado nutricional, segundo o IMC, classificadas em risco para sobrepeso, sobrepeso e obesidade, são 18 (21,95\%), em que 12 (14,63\%) são meninos e $6(7,31 \%)$ são meninas.

No estudo ${ }^{17}$ realizado na cidade de Londrina (PR), onde foram avaliados escolares da rede particular de ensino, foi detectado uma prevalência de $18,5 \%$ de sobrepeso e $13,4 \%$ de obesidade. Em outro trabalho, ${ }^{18}$ realizado em Marialva (PR) com alunos da rede municipal, foram identificados altos índices de sobrepeso (20\%) e obesidade (7\%); a diferença percentual da cidade de Londrina de sobrepeso e obesidade é maior que a de Marialva. Ambos os estudos foram diferentes dos nossos resultados, em que a somatória de risco para sobrepeso, sobrepeso e obesidade foi inferior a $22 \%$ de ambos os sexos.

Segundo o Instituto Brasileiro de Geografia e Estatística, ${ }^{19}$ estima-se que o número de crianças obesas no Brasil aumentou cinco vezes nos últimos vinte anos, atingindo atualmente $10 \%$ das crianças.

No entanto, vários são os fatores que levam os escolares a se tornarem obesos tais como aumento do tamanho da porção oferecida, alto consumo de alimentos calóricos, sedentarismo, falta de atividade física, a preferência por aparelhos eletrônicos (vídeo game, etc.) e grande oferta de alimentos industrializados, tudo isso, em conjunto com ambiente familiar influenciaram nas escolhas e padrões alimentares dos escolares.

O consumo alimentar dos escolares apresentou aspectos favoráveis e negativos em relação ao consumo dos alimentos saudáveis, houve diferença significativa entre as crianças com IMC adequado comparando-as com as que estão acima do peso, em que 52 (63,41\%) com IMC adequado comem mais de três vezes na semana, alimentos saudáveis como frutas e verduras. Independente do estado nutricional das crianças avaliadas, se estava adequado ou acima do peso, o consumo de leite e derivados e feijão foi diário.

Um estudo realizado em São Paulo, ${ }^{20}$ observou que o baixo consumo dos alimentos saudáveis como as frutas, verduras e legumes e o alto consumo de doces estavam associados ao sobrepeso e obesidade em escolares.

Para os alimentos não saudáveis não houve diferença significativa entre os escolares com IMC adequado, com aqueles que estão acima do peso. Este fato pode estar ligado ao elevado consumo dos alimentos não saudáveis (Tabela 2) e dentre eles podemos destacar muitos alimentos industrializados, tais como salgadinhos, biscoitos recheados, refrigerantes, etc. No estudo ${ }^{21}$ realizado com escolares matriculados em escolas de Melbourne (Austrália), encontraram associação significativa entre o hábito de assistir televisão, por um tempo igual ou maior que duas horas por dia e o consumo de petiscos salgados e bebidas altamente energéticas.

$\mathrm{Na}$ revisão sistemática, ${ }^{22}$ relacionou estudos que abordam a influência da televisão no consumo alimentar de criancas e adolescentes, em que o consumo dos alimentos não saudáveis é maior, quanto maior o tempo desperdiçado com a televisão. É provável que a interferência da televisão possa estar ligada à publicidade de alimentos não saudáveis, de baixo valor nutricional.

Os resultados deste estudo alertam para o cuidado com a alimentação e o estilo de vida que a família introduz aos seus filhos, sabendo-se que são diversos fatores que possam desencadear a obesidade, entre eles, a falta de atividade física e preferência alimentar. A escola representa um ambiente favorável para o desenvolvimento de estratégias que abordam assuntos como; bons hábitos alimentares e a importância da atividade física desde a infância até a fase adulta e, junto com o ambiente familiar, irá contribuir para um consumo de alimentos saudáveis ou não.

A prevenção é a melhor estratégica para o controle da obesidade, porém quando já instalada deve ser tratada com uma equipe multidisciplinar: médico, nutricionista, psicólogo e profissional de educação física, pois o excesso de peso interfere tanto no aspecto físico, quanto emocional do individuo. A família é fundamental para a prevenção ou tratamento, onde os filhos reproduzem o hábito alimentar dos pais. Novos estudos e estratégias são importantes para continuar avaliando a 
tendência de crescimento da prevalência da obesidade infantil e seus fatores associados.

\section{CONCLUSÃO}

Conclui-se que a maior parte das crianças avaliadas da rede municipal de ensino possuem IMC adequado e consomem alimentos saudáveis na maioria dos dias da semana, o que pode ser fator protetor para obesidade nesta população. Porém, há um percentual considerável acima do peso, o que requer cuidados e orientações especiais da escola e órgãos públicos de saúde e educação.

\section{REFERÊNCIAS}

1. WHO - World Health Organization. Report of a WHO Consultation on Obesity. Preventing and managing the global epidemic. WHO, Geneve, 1998.

2. IBGE - Instituto Brasileiro de Geografia e Estatística. POF 2008-2009 - Desnutrição cai e peso de crianças brasileiras ultrapassa padrão internacional, 2010. Disponível em: http://www.ibge.gov.br/home/estatistica/populacao/ condicaodevida/pof/2008_2009_aval_nutricional/ pof20082009_avaliacao.pdf

3. Pêgo-Fernandes PM, Bibas BJ, Deboni M. Obesidade: a maior epidemia do século XXI? São Paulo Med J 2011;129(5):283-4.

4. Abrantes, M.M.; Lamounier, J.A.; Colosimo, E.A. Prevalência de sobrepeso e obesidade nas regiões Nordeste e Sudeste do Brasil. Rev Assoc Med Bras 2003;49(2):162-166.

5. Halpern, A.; Mancini, M.C. Manual de Obesidade para o Clinico. São Paulo. Rocca. 2002.

6. Kiess W, Galler A, Reich A, Müller G, Kapellen T, Deutscher J, et al. Clinical aspects of obesity in childhood and adolescence. Obes Rev 2001;21(2):29-36. doi: 10.1046/j.1467-789x.2001.00017.x.

7. Francis LA, Lee $Y$, Birch LL. Parental weight status and girl's television viewing, snacking, and body mass index. Obes Res 2003;11:143-51. doi: 10.1038/oby.2003.23.

8. Singh AS, Mulder C, Twisk JWR, Van Mechelen W, Chinapaw MJM. Tracking of childhood overweight into adulthood: a systematic review of the literature. Obesity Rev 2008;9(5):47488. doi: 10.1111/j.1467-789X.2008.00475.x.

9. Carmo MB, Toral N, Silva MV, Slater B. Consumo de doces, refrigerantes e bebidas com adição de açúcar entre adolescentes da rede pública de ensino de Piracicaba, São Paulo. Rev Bra Epidemiol 2006;9(1):121:30. doi: 10.1590/ S1415-790X2006000100015.

10. Brasil - Ministério da Saúde. Secretaria da Atenção à Saúde. Guia alimentar para a população brasileira: promovendo a alimentação saudável. Brasília: Ministério da Saúde; 2006.

11. OMS- Organização Mundial de Saúde. Curvas de crescimento infantil. In: Brasil - Ministério da Saúde, 2007.

12. SISVAN- Sistema de Vigilância Alimentar e Nutricional. Protocolos do sistema de vigilância alimentar e nutricional. Brasília: Ministério da Saúde, 2008.

13. Stokes M E; Davis C S; Koth G G. Categorical data analysis using The SAS System; 2nd ed. SAS; 2009

14. Levine, D. M. et al. Estatística: teoria e aplicações usando MS-Excel. LTC -Livros Técnicos e Científicos; 1998.

15. WHO - World Health Organization. Physical status: the use and interpretation of anthropometry. Geneva: WHO, 1995. 452p. (Technical Report Series, 854).

16. Guedes DP. Recursos antropométricos para análise da composição corporal. Rev Bras Educ Fis Esp 2006;20:115-9.

17. Ronque ER, Cyrino ES, Dórea VR, Serassuelo Jr H, Galdi $\mathrm{EH}$, Arruda M. Prevalence of overweight and obesity in school children of socioeconomic level in Londrina, Paraná, Brazil. Rev Nutr 2005;18:709-17. doi: 10.1590/\$141552732005000600001 .

18. Mello ADM, Marcon SS, Hulsmeyer APCR, Cattai GBP, Ayres CSLS, Santana RG. Prevalência de sobrepeso e obesidade em crianças de seis a dez anos de escolas municipais de área urbana. Rev Paul Pedriatr 2010;28(1):48-54.

19. IBGE - Instituto Brasileiro de Geografia e Estatística. Pesquisa de orçamento familiares 2002-2003: antropometria e analise do estado nutricional de crianças e adolescente no Brasil. Rio de Janeiro, 2006.

20. Fagundes ALN, Ribeiro DC, Naspitz L, Garbelini LEB, Vieira JKP, Silva AP. Prevalência de sobrepeso e obesidade em escolares da região de Parelheiros do município de São Paulo. Rev Paul Pediatr 2008;26(3):212-7. doi 10.1590/S010305822008000300003.

21. Salmon J, Campbell KJ, Crawford DA. Television viewing habits associated with obesity risk factors: a survey of Melbourne schoolchildren. MJA 2006;182(2):64-7.

22. Rossi CE, Albernaz DO, Vasconcelos FAG, Assis MAA, Pietro PFD. A influência da televisão no consumo alimentar e na obesidade em crianças e adolescentes: uma revisão sistemática. Rev Nutr Campinas 2010;23(4):607-620. doi: 10.1590/S1415-52732010000400011.

Como citar: CADAMURO, Suelen Dayane Pereira et al. Consumo alimentar e avaliação nutricional: caracterização de escolares no município de Maringá - Paraná. Cinergis, Santa Cruz do Sul, v. 17, n. 2, jun. 2016. ISSN 2177-4005. Disponível em: <https://online.unisc.br/seer/index.php/cinergis/article/view/7764>. Acesso em: 04 jul. 2016. doi:http://dx.doi. org/10.17058/cinergis.v17i2.7764. 